\title{
NUMERICAL STUDIES OF VISCOUS INCOMPRESSIBLE FLOW BETWEEN TWO ROTATING CONCENTRIC SPHERES
}

\author{
E. O. IFIDON
}

Received 13 February 2004 and in revised form 12 March 2004

The problem of determining the induced steady axially symmetric motion of an incompressible viscous fluid confined between two concentric spheres, with the outer sphere rotating with constant angular velocity and the inner sphere fixed, is numerically investigated for large Reynolds number. The governing Navier-Stokes equations expressed in terms of a stream function-vorticity formulation are reduced to a set of nonlinear ordinary differential equations in the radial variable, one of second order and the other of fourth order, by expanding the flow variables as an infinite series of orthogonal Gegenbauer functions. The numerical investigation is based on a finite-difference technique which does not involve iterations and which is valid for arbitrary large Reynolds number. Present calculations are performed for Reynolds numbers as large as 5000. The resulting flow patterns are displayed in the form of level curves. The results show a stable configuration consistent with experimental results with no evidence of any disjoint closed curves.

\section{Introduction}

Confined rotating flows are usually solved using numerical techniques that involve iteration. This often reduces the region of applicability of the numerical method as a result of the instabilities arising from the iterative process on which the numerical method is based. In this paper, we apply a noniterative finite-difference technique to the study of the induced steady axially symmetric motion of an incompressible viscous fluid confined between two concentric spheres with the outer sphere rotating with constant angular velocity and the inner sphere fixed. The application of a new numerical technique to the rotating spheres problem is appropriate for the following reasons. First, the motion of viscous fluids in rotating containers is among the most fundamental problems in classical fluid dynamics and is of basic importance in finding a general hydrodynamic stability theory for rotating fluids in an enclosed cavity volume. Understanding the fluid dynamics of the motion is relevant in geophysical studies such as the study of atmospheric and oceanic circulations and also engineering design, particularly the study of 
gyroscopes and centrifuges. Second, the need to apply a new numerical technique to the rotating sphere problem is necessitated by the fact that there is a need to check the results in Greenspan [2], which differ substantially from those of Pearson [11], for $\operatorname{Re}=1000$. While Greenspan's result suggests the existence of two disjoint closed curves at the central portion of the flow region, Pearson's result does not predict the existence of such a phenomenon. Furthermore, Greenspan [2, Figure 14] shows the appearance of several disjoint closed curves for $\mathrm{Re}=3000$ while Schultz and Greenspan [13, Figure 6] show that there are only two sets of closed curves in the central region. Finally, researchers are always interested in numerical methods that are accurate and stable for a wide range of flow parameters. Many papers have been published on the numerical solution of steady, viscous, incompressible flow between two rotating concentric spheres. However, these papers have used iterative techniques and have produced results only for $\operatorname{Re} \leq 3000$. We present a noniterative method which is valid for both small and large Reynolds number and obtain results as large as $\mathrm{Re}=5000$.

\section{Governing equations of motion}

We consider the motion of an isothermal incompressible viscous fluid contained in a spherical annulus between two rotating concentric spheres. The spheres are assumed to be rigid and rotate freely about a vertical axis through their centre, which is taken to be the origin of the coordinate system. The inner sphere and outer sphere are constrained to rotate with prescribed angular velocities $\omega_{1}$ and $\omega_{2}$, respectively. We start with the full incompressible Navier-Stokes equation with no body force [14],

$$
\frac{\partial \vec{q}}{\partial t}+\vec{q} \vec{\nabla} \cdot \vec{q}=-\vec{\nabla} p+\frac{1}{\operatorname{Re}} \nabla^{2} \vec{q}
$$

together with the incompressibility condition,

$$
\vec{\nabla} \cdot \vec{q}=0
$$

and the no-slip (rigid) boundary conditions on the spherical surfaces,

$$
\begin{array}{ll}
\vec{q}=r \omega_{1} \sin \theta \hat{e}_{\varphi} & \text { at } r=r_{1}, \\
\vec{q}=r \omega_{2} \sin \theta \hat{e}_{\varphi} & \text { at } r=r_{2},
\end{array}
$$

where $\vec{q}=(u, v, w)$ is the velocity field, $p$ is the kinematic pressure, and Re is the Reynolds number defined by $\omega_{0} r_{2}^{2} / v$. In the two extreme cases, $\omega_{0}=\omega_{1}$ if the outer sphere is stationary and $\omega_{0}=\omega_{2}$ if the inner sphere is stationary. When both spheres rotate, the character of the flow is generally dominated by the motion of either the inner or outer sphere. The angular velocity of the dominant sphere is then taken as the characteristic angular velocity. The specific choice of $\omega_{0}$ is indicated for each example considered. The governing equations have been nondimensionalized with the characteristic velocity scale $\omega_{0} r_{2}$ and the characteristic length scale $r_{2}$. If the spherical polar coordinates $(r, \theta, \varphi)$ are chosen with the origin at the centre of the sphere and $\theta=0$, the axis of rotation, the motion is 
independent of the azimuthal angle $\varphi$ and the Navier-Stokes equations can then be written in terms of a stream function in the meridian plane, $\Psi$, an angular velocity function $\Omega$, as well as a vorticity function $\Phi$. Thus, making the substitutions,

$$
u=\frac{\Psi_{\theta}}{r^{2} \sin \theta}, \quad v=-\frac{\Psi_{r}}{r \sin \theta}, \quad w=\frac{\Omega}{r \sin \theta}, \quad(\vec{\nabla} \times \vec{q}) \cdot \hat{e}_{\varphi}=\frac{1}{r \sin \theta} \Phi,
$$

the Navier-Stokes equations (2.1) and (2.2) are easily found to be

$$
\begin{gathered}
E^{2} \Omega=\frac{\operatorname{Re}}{r^{2} \sin \theta}\left[\Psi_{\theta} \Omega_{r}-\Psi_{r} \Omega_{\theta}\right], \\
E^{2} \Psi=\Phi, \\
E^{2} \Phi=\operatorname{Re}\left\{\frac{2 \Omega}{r^{3} \sin ^{2} \theta}\left[\Omega_{r} r \cos \theta-\Omega_{\theta} \sin \theta\right]+\frac{1}{r^{2} \sin \theta}\left[\Psi_{\theta} \Phi_{r}-\Psi_{r} \Phi_{\theta}\right]\right. \\
\left.+\frac{2 \Phi}{r^{3} \sin ^{2} \theta}\left[\Psi_{r} r \cos \theta-\Psi_{\theta} \sin \theta\right]\right\}=0,
\end{gathered}
$$

where

$$
E^{2}=\frac{\partial^{2}}{\partial r^{2}}+\frac{1}{r^{2}} \frac{\partial^{2}}{\partial \theta^{2}}-\frac{1}{r^{2}} \cot \theta \frac{\partial}{\partial \theta}
$$

In view of the boundary conditions, we may assume the expansions

$$
\begin{aligned}
& \Omega(\mu, x)=\sum_{n=1}^{\infty} I_{2 n}(\mu) W_{n}(x), \\
& \Psi(\mu, x)=\sum_{n=1}^{\infty} I_{2 n+1}(\mu) \psi_{n}(x), \\
& \Phi(\mu, x)=\sum_{n=1}^{\infty} I_{2 n+1}(\mu) Y_{n}(x),
\end{aligned}
$$

where $I_{n}(\mu)$ are the Gegenbauer functions of argument $\mu=\cos \theta$. These functions form a complete orthogonal set in the range $-1 \leq \mu \leq 1$. We have substituted $r=e^{-x}$. Substituting (2.9)-(2.11) in the equations (2.5)-(2.8) and making use of the orthogonality properties of the functions $I_{n}(\mu)$ give rise to infinite sets of ordinary differential equations. Substitution into $(2.5)$ yields

$$
\frac{d^{2} W_{n}}{d x^{2}}+\frac{d W_{n}}{d x}-2 n(2 n-1) W_{n}=\sum_{i=1}^{\infty} A_{3}(x) \frac{d W_{i}}{d x}+\sum_{i=1}^{\infty} A_{4}(x) W_{i},
$$

where

$$
\begin{aligned}
& A_{3}(x)=-\operatorname{Re} \sum_{l=1}^{\infty} e^{x} \psi_{l}(x) d_{1}(2 n, 2 i, 2 l+1), \\
& A_{4}(x)=\operatorname{Re} \sum_{l=1}^{\infty} e^{x} \psi_{l}^{\prime}(x) d_{1}(2 n, 2 l+1,2 i) .
\end{aligned}
$$


94 Viscous flow between rotating concentric spheres

By the use of similar procedures, (2.6) and (2.7) may be reduced to the respective sets of equations

$$
\begin{aligned}
& \frac{d^{2} \psi_{n}}{d x^{2}}+\frac{d \psi_{n}}{d x}-2 n(2 n+1) \psi_{n}=e^{-2 x} Y_{n}(x), \\
& \frac{d^{2} Y_{n}}{d x^{2}}+\frac{d Y_{n}}{d x}-2 n(2 n+1) Y_{n}=R_{n}(x),
\end{aligned}
$$

where $R_{n}(x)$ are nonlinear combinations of the various functions defined by

$$
\begin{aligned}
R_{n}(x)= & \sum_{i=1}^{\infty} A_{3}(x) \frac{d Y_{i}}{d x}+\sum_{i=1}^{\infty} A_{4}(x) Y_{i} \\
& +\operatorname{Re} \sum_{i=1}^{\infty} \sum_{l=1}^{\infty} e^{x}\left\{W_{i}(x) W_{l}^{\prime}(x) d_{2}(2 n+1,2 l, 2 i)-2 W_{i}(x) W_{l}(x) d_{1}(2 n+1,2 i, 2 l)\right\} \\
A_{3}(x)= & -\operatorname{Re} \sum_{l=1}^{\infty} e^{x} \psi_{l}(x) d_{1}(2 n+1,2 i+1,2 l+1), \\
A_{4}(x)= & \operatorname{Re} \sum_{l=1}^{\infty} e^{x}\left[\psi_{l}^{\prime}(x)\left\{d_{1}(2 n+1,2 i+1,2 l+1)-d_{2}(2 n+1,2 l+1,2 i+1)\right\}\right. \\
& \left.\quad-2 \psi_{l}(x) d_{1}(2 n+1,2 i+1,2 l+1)\right] .
\end{aligned}
$$

The quantities $d_{1}(n, m, k)$ and $d_{2}(n, m, k)$ which appear in (2.13), (2.17), and (2.18) are integrals involving products of Gegenbauer functions and derived functions and are given by

$$
\begin{aligned}
d_{1}(n, m, k) & =\frac{n(n-1)(2 n-1)}{2} \int_{-1}^{1} \frac{I_{n}(\mu) I_{m}(\mu) P_{k-1}(\mu)}{1-\mu^{2}} d \mu \\
& =\sum_{i=0}^{n-2 i-2 \geq 0} \frac{(2 n-1)(2 n-4 i-3)}{2(2 m-1)} \int_{-1}^{1} P_{k-1}(\mu) P_{n-2 i-2}(\mu)\left\{P_{m-2}(\mu)-P_{m}(\mu)\right\} d \mu, \\
d_{2}(n, m, k) & =(n-1)(n)(2 n-1) \int_{-1}^{1} \frac{\mu I_{n}(\mu) I_{m}(\mu) I_{k}(\mu)}{\left(1-\mu^{2}\right)^{2}} d \mu \\
& =\frac{2 d_{1}(n, m, k)}{k}+\sum_{i=0}^{k-2 i-3 \geq 0} \frac{2(2 k-2 i-5)}{k(k-1)} d_{1}(n, m, k-2 i-2) .
\end{aligned}
$$

The boundary conditions corresponding to (2.12) are

$$
W_{n}\left(c_{1}\right)=2 h_{1} b_{1}^{2} \delta_{1 n}, \quad W_{n}\left(c_{2}\right)=2 h_{2} b_{2}^{2} \delta_{1 n},
$$


where $c_{i}=-\log _{e} b_{i}, b_{i}=r_{i} / r_{2}$ are the surfaces of the spheres, $h_{i}=\omega_{i} / \omega_{0}$, and $\delta_{n, 1}$ is the Kronecker delta function. The boundary conditions for (2.14) are

$$
\psi_{n}\left(c_{1}\right)=\psi_{n}\left(c_{2}\right)=\psi_{n}^{\prime}\left(c_{1}\right)=\psi_{n}^{\prime}\left(c_{2}\right)=0 .
$$

The functions $Y_{n}(x)$ are not known at the surface of the sphere and can be calculated as part of the solution. One way of doing this is to multiply (2.14) by $e^{-(2 n+1) x}$ and $e^{2 n x}$ in turn, then integrate both sides of the equation with respect to $x$ from $c_{1}$ to $c_{2}$. After some integration by parts of the terms on the left-hand side and the use of the conditions (2.21), we have

$$
\begin{aligned}
& \int_{c_{1}}^{c_{2}} e^{-(2 n+2) x} Y_{n}(x) d x=0, \\
& \int_{c_{1}}^{c_{2}} e^{-(2 n-1) x} Y_{n}(x) d x=0 .
\end{aligned}
$$

The use of (2.22) poses additional computational difficulties as their values need to be determined along with the other flow variables during the computational process. Conditions (2.22) act as an initial guess for the boundary conditions for $Y_{n}(x)$ and are to be improved upon iteratively during the computational process. In principle, any quadrature formulae may be used but in practice; care needs to be taken about the choice of quadrature used as this may lead to inaccuracies and subsequent instability of the overall computational process. Since we are interested in developing a computational procedure that does not involve any iteration, at least in the conventional sense, it would be computationally more advantageous if the fourth-order equation is considered instead. Thus we substitute $\psi_{n}(x)=e^{-2 x} S_{n}(x)$, so that (2.14) becomes

$$
Y_{n}(x)=\frac{d^{2} S_{n}}{d x^{2}}+3 \frac{d S_{n}}{d x}+(2-2 n(2 n+1)) S_{n}
$$

and (2.15) becomes

$$
\left[\frac{d^{2}}{d x^{2}}+\frac{d}{d x}-2 n(2 n+1)\right]\left[\frac{d^{2}}{d x^{2}}-3 \frac{d}{d x}+(2-2 n(2 n+1))\right] S_{n}(x)=R_{n}(x),
$$

where

$$
\begin{aligned}
R_{n}(x)= & \sum_{i=1}^{\infty} A_{3}(x)\left[\frac{d^{3}}{d x^{3}}-3 \frac{d^{2}}{d x^{2}}+(2-2 i(2 i+1)) \frac{d}{d x}\right] S_{i}(x) \\
& +\sum_{i=1}^{\infty} A_{4}(x)\left[\frac{d^{2}}{d x^{2}}-3 \frac{d}{d x}+(2-2 i(2 i+1))\right] S_{i}(x) \\
& +\operatorname{Re} \sum_{i=1}^{\infty} \sum_{l=1}^{\infty} e^{x}\left\{W_{i}(x) W_{l}^{\prime}(x) d_{2}(2 n+1,2 l, 2 i)-2 W_{i}(x) W_{l}(x) d_{1}(2 n+1,2 i, 2 l)\right\} .
\end{aligned}
$$

The boundary conditions imposed on $S_{n}(x)$ are

$$
S_{n}\left(c_{1}\right)=S_{n}\left(c_{2}\right)=S_{n}^{\prime}\left(c_{1}\right)=S_{n}^{\prime}\left(c_{2}\right)=0
$$


In the practical numerical integrations, the computations must be limited to the determination of a finite number of terms $n s$ in each of the series (2.9), (2.10), and (2.11) by truncation of the series. We will determine later an appropriate choice for $n s$, for which terms with subscript greater than $n s$ will be set to zero.

\section{Computational method}

Before we embark on the discretization of the governing equations in the computational domain between the two concentric spheres by a suitable finite-difference method, we observe from the previous numerical experiments that in finding numerical solutions to viscous incompressible flow problems, one discovers that for high Reynolds numbers, that is, $\operatorname{Re}>400$, one can use the solutions for lower Reynolds number flow as a starting point in an iterative process to get solutions for higher values of Re. Convergence of the overall iterative process may not be achieved using other starting values. Thus the results of the computations are quite sensitive to the choice of the initial guess approximations. This suggests that one can obtain a relationship between the initial guess and the final solution. This is possible using homotopy methods. Homotopy, continuation, or embedding methods are well known and have been used in the past for finding zeros of nonlinear functions [1]. Recently, the method has been given renewed attention and it is being used for the computation of matrix eigenvalues and eigenvectors [10] as well as for obtaining analytic solutions to nonlinear partial differential equations (p.d.e) $[3,4]$. The basic idea is to construct a homotopy or mapping from a trivial or known solution (initial guess) to the desired solution. This is achieved by first defining a homotopy or deformation in an embedding parameter $\lambda \in[0,1]$ of the form

$$
H: R^{n} \times[0,1] \longrightarrow R^{n}
$$

such that

$$
H(x, 0)=G(x), \quad H(x, 1)=F(x),
$$

where $G: R^{n} \rightarrow R^{n}$ is a trivial or known solution and $H$ is assumed to be smooth. Typically, one chooses a homotopy such that

$$
H(x, \lambda):=(1-\lambda) G(x)+\lambda F(x)
$$

and attempts to trace an implicitly defined curve $c(s)$ in the parametric set of this homotopy map from a starting point to a solution point. In order to ensure that this curve intersects the homotopy level $\lambda=1$ at finite points, it is sufficient to impose suitable boundary conditions which essentially prevent the curve from running to infinity before intersecting the homotopy level $\lambda=1$ or from returning to the initial level. This basic concept may be extended to a system of nonlinear p.d.e.

$$
\Delta F(x)=0
$$

in a natural way. The idea is to start with a problem $\Delta_{0} G(x)=0$ with known or trivial solutions, then to continuously transform its solutions to those of the original problem (3.4). These solutions are the continuous functions corresponding to a family of 
differential operators $\Delta(x, \lambda), \lambda \in[0,1]$, with

$$
\Delta(x, 0)=\Delta_{0}, \quad \Delta(x, 1)=\Delta
$$

given by

$$
\Delta(x, \lambda):=(1-\lambda) \Delta_{0}+\lambda \Delta .
$$

The starting problem $\Delta_{0} G(x)=0$ should meet two requirements. First, its solutions $G(x)$ should be easy to compute. This is obviously best met by a p.d.e whose solutions are already known. Second, its solutions must satisfy the same initial and boundary conditions as those of the original problem. The corresponding homotopy equation can easily be derived from (3.4) by replacing $\Delta$ with $\Delta(x, \lambda)$ and $F(x)$ with $H(x, \lambda)$. Thus the homotopy equation is

$$
\Delta(x, \lambda) H(x, \lambda)=0
$$

or, using (3.6),

$$
(1-\lambda) \Delta_{0} H(x, \lambda)+\lambda \Delta H(x, \lambda)=0 .
$$

We see from (3.8) that the process of $\lambda$ increasing from zero to one is just the process of $H$ going from $G(x)$, the initial guess approximation, to $F(x)$, the desired solution. This process is called deformation in topology. Equation (3.8) would be referred to as the zeroth-order deformation equation. We apply this concept of homotopy to the solutions of (2.12) and (2.24). For (2.12), we start with the linear problem

$$
\Delta_{0} \Omega_{n} \equiv Q(x)\left[\frac{d^{2}}{d x^{2}}+\frac{d}{d x}-2 n(2 n-1)\right] \Omega_{n}(x)=0
$$

subject to the boundary conditions

$$
\Omega_{n}\left(c_{1}\right)=2 h_{1} b_{1}^{2} \delta_{1 n}, \quad \Omega_{n}\left(c_{2}\right)=2 h_{2} b_{2}^{2} \delta_{1 n}
$$

where $Q(x)$ is a nonzero arbitrary real-valued function. We then go on to solve the nonlinear equation

$$
\Delta W_{n} \equiv\left[\frac{d^{2}}{d x^{2}}+\frac{d}{d x}-2 n(2 n-1)\right] W_{n}(x)-\left[\sum_{i=1}^{\infty} A_{3}(x) \frac{d}{d x}+\sum_{i=1}^{\infty} A_{4}(x)\right] W_{i}(x)=0
$$

subject to (2.20). Observe that $Q(x)$ completely determines $\Delta_{0}$ and its selection becomes very important. We construct a family of ordinary differential equations in an embedding parameter $\lambda$ such that

$$
(1-\lambda) \Delta_{0} W_{n}(x, \lambda)+\lambda \Delta W_{n}(x, \lambda)=0
$$


with the corresponding boundary conditions

$$
W_{n}\left(c_{1}, \lambda\right)=2 h_{1} b_{1}^{2} \delta_{1 n}, \quad W_{n}\left(c_{2}, \lambda\right)=2 h_{2} b_{2}^{2} \delta_{1 n} .
$$

When $\lambda=0$, we see from (3.12) and (3.9) that

$$
W_{n}(x, 0)=\Omega_{n}(x) .
$$

Also, when $\lambda=1$, we see from (3.12) and (3.11) that

$$
W_{n}(x, 1)=W_{n}(x) .
$$

Thus the process of $\lambda$ increasing from zero to one is just the process of $W_{n}$ varying from $\Omega_{n}(x)$ to $W_{n}(x)$. Obviously, (3.14) and (3.15) give a relationship between the initial guess $\Omega_{n}(x)$ and the solution $W_{n}(x)$. However, this kind of relationship is indirect; a direct relationship between $\Omega_{n}(x)$ and $W_{n}(x)$ must be obtained. Following [3, 4], we assume that the deformations $W_{n}(x, \lambda)$ are smooth enough about $\lambda=0$ so that all of the $m$ thorder deformation derivatives

$$
\Omega_{n}^{[m]}(x)=\left.\frac{\partial^{m} W_{n}(x, \lambda)}{\partial \lambda^{m}}\right|_{\lambda=0}, \quad m \geq 1,
$$

exist, then expanding $W_{n}(x, \lambda)$ in a Taylor series about $\lambda=0$ in the closed interval $[0,1]$, we have

$$
W_{n}(x, \lambda)=W_{n}(x, 0)+\sum_{m=1}^{\infty} \frac{\Omega_{n}^{[m]}(x)}{m !} \lambda^{m} .
$$

Writing

$$
W_{m, n}(x)=\frac{\Omega_{n}^{[m]}(x)}{m !},
$$

we have from (3.14) that

$$
W_{n}(x, \lambda)=\Omega_{n}(x)+\sum_{m=1}^{\infty} W_{m, n}(x) \lambda^{m} .
$$

Assuming the above Taylor series is convergent at $\lambda=1$, we have from (3.15) that

$$
W_{n}(x)=\Omega_{n}(x)+\sum_{m=1}^{\infty} W_{m, n}(x) .
$$

The above expression (3.20) gives a direct relationship between the initial guess approximations $\Omega_{n}(x)$ and the required solution $W_{n}(x)$ through the unknown terms $W_{m, n}(x)$ whose defining equation can be obtained by differentiating the zeroth-order deformation 
equations (3.12) $m$ times with respect to $\lambda$, then setting $\lambda=0$, and finally dividing it by $m$ !. Thus

$$
\Delta_{0} W_{m, n}(x)=q_{m, n}(x), \quad m \geq 1,
$$

with the boundary conditions

$$
W_{m, n}\left(c_{1}\right)=W_{m, n}\left(c_{2}\right)=0, \quad m, n \geq 1
$$

The right-hand side term $q_{m, n}(x)$ is given by

$$
\begin{aligned}
q_{m, n}(x)= & \left(\chi_{m} \Delta_{0}-\left(\frac{d^{2}}{d x^{2}}+\frac{d}{d x}-2 n(2 n-1)\right)\right) W_{m-1, n}(x) \\
& +\left(\sum_{i=0}^{\infty} \sum_{k=0}^{m-1} A_{3, k} \frac{d}{d x} W_{m-1-k, i}(x)\right)+\sum_{i=1}^{\infty} \sum_{k=0}^{m-1} A_{4, k} W_{m-1-k, i}(x)
\end{aligned}
$$

and the coefficient $\chi_{m}$ is defined by

$$
\chi_{m}= \begin{cases}0 & \text { when } m \leq 1 \\ 1 & \text { when } m \geq 2\end{cases}
$$

Equation (3.21) is called the $m$ th-order deformation equations. It should be emphasized that all of the above $m$ th-order deformation equations are linear differential equations. Thus, through this approach, one solves an infinite sequence of linear subproblems instead of the original nonlinear equations (3.11). The initial guess approximation can be obtained from (3.9) and (3.10) given the value of $Q(x)$. Neglecting the nonlinear terms in (2.24) should give us the desired initial guess for (2.24). However, the last nonlinear term on the right-hand side of (2.24) provides the "driving force" for this equation and so cannot be neglected. One can therefore start with the problem

$$
\begin{aligned}
\Delta_{0}\left(\phi_{n}(x), \Omega_{n}(x)\right) \equiv Z(x)\{ & {\left[\frac{d^{2}}{d x^{2}}+\frac{d}{d x}-2 n(2 n+1)\right] } \\
& \left.\times\left[\frac{d^{2}}{d x^{2}}-3 \frac{d}{d x}+(2-2 n(2 n+1))\right] \phi_{n}(x)-f_{n}(x)\right\},
\end{aligned}
$$

where $Z(x)$ is an arbitrary nonzero real-valued function and

$$
f_{n}(x)=\operatorname{Re} \sum_{i=1}^{\infty} \sum_{l=1}^{\infty} e^{x}\left\{\Omega_{i}(x) \frac{d \Omega_{j}}{d x} d_{2}(2 n+1,2 l, 2 i)-2 \Omega_{i}(x) \Omega_{l}(x) d_{1}(2 n+1,2 i, 2 l)\right\}
$$

with boundary conditions

$$
\phi_{n}\left(c_{1}\right)=\phi_{n}\left(c_{2}\right)=\frac{d \phi_{n}}{d x}\left(c_{1}\right)=\frac{d \phi_{n}}{d x}\left(c_{2}\right)=0
$$


then go on to solve the nonlinear problem

$$
\begin{aligned}
& \Delta\left(S_{n}(x), W_{n}(x)\right) \\
& \quad \equiv\left[\frac{d^{2}}{d x^{2}}+\frac{d}{d x}-2 n(2 n+1)\right]\left[\frac{d^{2}}{d x^{2}}-3 \frac{d}{d x}+(2-2 n(2 n+1))\right] S_{n}(x)-R_{n}(x),
\end{aligned}
$$

where

$$
\begin{aligned}
R_{n}(x)= & \sum_{i=1}^{\infty} A_{3}(x)\left[\frac{d^{3}}{d x^{3}}-3 \frac{d^{2}}{d x^{2}}+(2-2 i(2 i+1)) \frac{d}{d x}\right] S_{i}(x) \\
& +\sum_{i=1}^{\infty} A_{4}(x)\left[\frac{d^{2}}{d x^{2}}-3 \frac{d}{d x}+(2-2 i(2 i+1))\right] S_{i}(x) \\
& +\operatorname{Re} \sum_{i=1}^{\infty} \sum_{l=1}^{\infty} e^{x}\left\{W_{i}(x) \frac{d W_{j}}{d x} d_{2}(2 n+1,2 l, 2 i)-2 W_{i}(x) W_{l}(x) d_{1}(2 n+1,2 i, 2 l)\right\}
\end{aligned}
$$

subject to the boundary conditions (2.26). The choice of $Z(x)$ is very important as it completely determines $\Delta_{0}$. The zeroth-order deformation equation in an embedding parameter $\lambda$ is then

$$
(1-\lambda) \Delta_{0}\left(\Psi_{n}(x, \lambda), \Omega_{n}(x)\right)+\lambda \Delta\left(\Psi_{n}(x, \lambda), W_{n}(x, \lambda)\right)=0
$$

with the corresponding boundary conditions

$$
\Psi_{n}\left(c_{1}, \lambda\right)=\Psi_{n}\left(c_{2}, \lambda\right)=\left.\frac{d}{d x} \Psi_{n}(x, \lambda)\right|_{x=c_{1}}=\left.\frac{d}{d x} \Psi_{n}(x, \lambda)\right|_{x=c_{2}}=0 .
$$

When $\lambda=0$, we see from (3.30) that

$$
\Psi_{n}(x, 0)=\phi_{n}(x)
$$

When $\lambda=1$, we see from (3.30) that

$$
\Psi_{n}(x, 1)=S_{n}(x) .
$$

Thus, as $\lambda$ increases from zero to one, $\Psi_{n}(x, \lambda)$ increases from the initial guess approximation to the desired solution $S_{n}(x)$. Therefore, (3.31) and (3.32) give a relationship between the initial guess $\phi_{n}(x)$ and the solution $S_{n}(x)$. However, this kind of relationship is indirect. A direct relationship between $\phi_{n}(x)$ and $S_{n}(x)$ can be obtained as follows. Assume that the deformations $\Psi_{n}(x, \lambda)$ are smooth enough about $\lambda=0$ so that all of the $m$ th-order deformation derivatives

$$
\phi_{n}^{[m]}(x)=\left.\frac{\partial^{m} \Psi_{n}(x, \lambda)}{\partial \lambda^{m}}\right|_{\lambda=0}, \quad m \geq 1,
$$

exist, then, by Taylor series, we have

$$
\Psi_{n}(x, \lambda)=\Psi_{n}(x, 0)+\sum_{m=1}^{\infty} \frac{\phi_{n}^{[m]}(x)}{m !} \lambda^{m} .
$$


Writing

$$
\psi_{m, n}(x)=\frac{\phi_{n}^{[m]}(x)}{m !},
$$

we have from (3.32) that

$$
\Psi_{n}(x, \lambda)=\phi_{n}(x)+\sum_{m=1}^{\infty} \psi_{m, n}(x) \lambda^{m} .
$$

Assuming the above Taylor series is convergent at $\lambda=1$, we have from (3.33) that

$$
S_{n}(x)=\phi_{n}(x)+\sum_{m=1}^{\infty} \psi_{m, n}(x) .
$$

The above expression gives a direct relationship between the initial guess approximation $\phi_{n}(x)$ and the desired solution $S_{n}(x)$ through the unknown terms $\psi_{m, n}(x)$ whose governing equation can be obtained from (3.30) by differentiating the zeroth-order deformation equation (3.30) $m$ times with respect to $\lambda$, then setting $\lambda=0$, and finally dividing by $m$ !. Thus we have

$$
\Delta_{m, n} \psi_{m, n}(x)=h_{m, n}(x), \quad m \geq 1
$$

where the linear differential operator $\Delta_{m, n}$ is given as

$$
\Delta_{m, n}=Z(x)\left[\frac{d^{2}}{d x^{2}}+\frac{d}{d x}-2 n(2 n+1)\right]\left[\frac{d^{2}}{d x^{2}}-3 \frac{d}{d x}+(2-2 n(2 n+1))\right]
$$

and the right-hand side term $h_{m, n}(x)$ is defined as

$$
\begin{aligned}
h_{m, n}(x)= & {\left[\chi_{m} \Delta_{m, n}-\left(\frac{d^{2}}{d x^{2}}+\frac{d}{d x}-2 n(2 n+1)\right)\right.} \\
& \left.\times\left(\frac{d^{2}}{d x^{2}}-3 \frac{d}{d x}+(2-2 n(2 n+1))\right)\right] \psi_{m-1, n}(x) \\
& +\sum_{i=1}^{\infty} \sum_{k=0}^{m-1} A_{3, k}\left(\frac{d^{3}}{d x^{3}}-3 \frac{d^{2}}{d x^{2}}+(2-2 i(2 i+1)) \frac{d}{d x}\right) \psi_{m-1-k, i}(x) \\
& +\sum_{i=1}^{\infty} \sum_{k=0}^{m-1} A_{4, k}\left(\frac{d^{2}}{d x^{2}}-3 \frac{d}{d x}+(2-2 i(2 i+1))\right) \psi_{m-1-k, i}(x) \\
& -\operatorname{Re} \sum_{i=1}^{\infty} \sum_{j=1}^{\infty} \sum_{k=0}^{m-1} e^{x}\left[\left(\frac{d}{d x} W_{k, j}\right) d_{2}(2 n+1,2 j, 2 i)\right. \\
& \left.-2 W_{k, l} d_{1}(2 n+1,2 i, 2 j)\right] W_{m-1-k, i}(x),
\end{aligned}
$$


where

$$
\begin{aligned}
A_{3, k}(x)=-\operatorname{Re} \sum_{l=1}^{\infty} e^{-x} \psi_{k, l}(x) d_{1}(2 n+1,2 i+1,2 l+1) \\
A_{4, k}(x)=\operatorname{Re} \sum_{l=1}^{\infty} e^{-x}\left[\left(-2 \psi_{k, l}(x)-\frac{d}{d x} \psi_{k, l}(x)\right)\right. \\
\times\left(d_{1}(2 n+1,2 i+1,2 l+1)-d_{2}(2 n+1,2 l+1,2 i+1)\right. \\
\left.\left.-2 \psi_{k, l}(x) d_{1}(2 n+1,2 i+1,2 l+1)\right)\right]
\end{aligned}
$$

with the corresponding boundary conditions

$$
\psi_{m, n}\left(c_{1}\right)=\psi_{m, n}\left(c_{2}\right)=\left.\frac{d}{d x} \psi_{m, n}(x)\right|_{x=c_{1}}=\left.\frac{d}{d x} \psi_{m, n}(x)\right|_{x=c_{2}}=0
$$

The basic problem now is to determine a suitable choice for $Q(x)$ as well as for $Z(x)$, then to go on to solve the set of ordinary differential equations (3.21) and (3.39) subject to conditions (3.22) and (3.43) using an appropriate finite-difference method. In the practical numerical integration of these equations, the computations must be limited to the determination of a finite number of terms in each of the series (2.9), (2.10), and (2.11) by truncation of the series. A truncation of order $n s$ is defined to be the process of setting to zero all the terms with subscript greater than $n s$ in (2.9), (2.10), and (2.11) and likewise in (2.12) and (2.25) and solving the resulting set of $2 n s$ differential equations subject to the prescribed boundary conditions. Also, in the practical evaluation of the series (3.20) and (3.38), we assume we have summed up enough terms when the term we have just added is smaller than some small $\varepsilon$ times the magnitude of the sum thus far accumulated. If the terms do not get small fast enough, the series becomes unsuitable for numerical computations. The convergence of a series such as (3.20) and (3.38) as given by the homotopy analysis method depends upon the choice of the initial approximation and the choice of the auxiliary linear operator and the auxiliary functions $Q(x), Z(x)$. As long as the initial approximation is not too bad, one can get convergent results by choosing a proper value for $Q(x), Z(x)$, although a worse initial approximation often corresponds to a slowly convergent series. There are many ways to choose a best initial approximation; also, one can apply a generalized Taylor theorem to greatly enlarge convergence regions of approximation series (3.20) and (3.38) [5,6].

The numerical method uses a novel form of finite-difference representations, which involves exponential terms and is superior to standard truncated Taylor representation and which was originally developed by Allen and Southwell for second-order ordinary differential equations [12]. The finite-difference representations of the fourth-order equation (3.39) as well as the second-order equations (3.21) are derived following a procedure that involves considering the coefficients of the differential equations as constant coefficient equations and obtaining for each equation an exact form of difference equations (exact in the sense that if the equations were constant coefficient ordinary differential 
equations, then the solution of the difference equations would be exact). Using this approach, the finite-difference scheme corresponding to (3.21) can then be written as

$$
\begin{gathered}
e^{-(2 n-i) h} W_{m, n, k+1}-\left(1+e^{-(4 n-1) h}\right) W_{m, n, k}+e^{-2 n h} W_{m, n, k-1} \\
=\frac{\left(1-e^{-(2 n-1) h}\right)\left(1-e^{-2 n h}\right)}{2 n(2 n-1)} q_{m, n}\left(x_{k}\right),
\end{gathered}
$$

while the finite-difference scheme corresponding to (3.39) is

$$
\begin{aligned}
&-e^{-h} \psi_{m, n, k+2}+\left(e^{(2 n-1) h}+e^{-(2 n+2) h}+e^{-2 n h}+e^{(2 n+1) h}\right) \psi_{m, n, k+1} \\
&-\left(2+e^{-2 h}+e^{(4 n+1) h}+e^{-(4 n+1) h}+e^{2 h}\right) \psi_{m, n, k} \\
&+\left(e^{2 n h}+e^{(2 n+2) h}+e^{-(2 n-1) h}+e^{-(2 n-1) h}+e^{-(2 n+1) h}\right) \psi_{m, n, k-1}-e^{h} \psi_{m, n, k-2} \\
&= \frac{\left(-1+e^{2 n h}\right)\left(1-e^{-(2 n+2) h}\right)\left(1-e^{-(2 n-1) h}\right)\left(1-e^{(2 n+1) h}\right)}{(2 n+1)(2 n(2 n+1)-2)} h_{m, n}(x) .
\end{aligned}
$$

A standard tridiagonal matrix algorithm (TDMA) can be used to evaluate (3.44) and a modified TDMA is used to evaluate (3.45). An implementation of the TDMA is given in [15].

\section{Results and discussion}

The calculations are carried out over a range of Re from 10 to 5000. Truncations of higher order were carried out for a given value of Re by using the results of the previous truncation as a starting assumption. In this way, the number of terms in the solution was built up. For small Re, convergence was quite rapid and only a few terms were required to describe the properties of the solution quite accurately. As the Reynolds number is increased, the number of terms required to give good accuracy also increases. Solutions were carried out for several values of $n s$ for each value of Re and some judgment was made as to the maximum value of $n s$ required. As Re increases, more terms are required for convergence. It was found that the initial guess approximation for $\operatorname{Re}=100 \mathrm{can}$ also be used as a starting value for higher values of Re. A suitable choice of $Q(x)$ and $Z(x)$ needs to be made to ensure convergence of the series (3.20) and (3.38), respectively. Typically, one chooses $Q(x)=Z(x)=1$ for values of $\operatorname{Re}<1000$ and $Z(x)=\operatorname{Re}$ for higher values. A close examination of the computer output shows that, in the exception of end points, the first term used in the series representation of the stream functions, namely, $S_{1}(x)$, is positive definite and other terms, namely, $S_{n}(x), n>1$, can change sign. Also, we observe that for the cases considered, $S_{1}(x) \gg\left|S_{n s}(x)\right|$. This ensures the overall convergence of our computed solutions. The radius of the inner sphere was taken as half that of the outer sphere. The essence of this is to check the results of Greenspan [2] as well as Schultz and Greenspan [13] which differ substantially from those of Pearson [11] for $\operatorname{Re}=1000$. A plot of the stream functions for $\mathrm{Re}<1000$ agrees qualitatively with those of Pearson and are consistent with the results of Munson and Joseph [7, 8] and other published results. Secondary flow patterns were clearly visible in the meridional plane and a recirculation region developed near the equator. It was observed that the secondary flow 


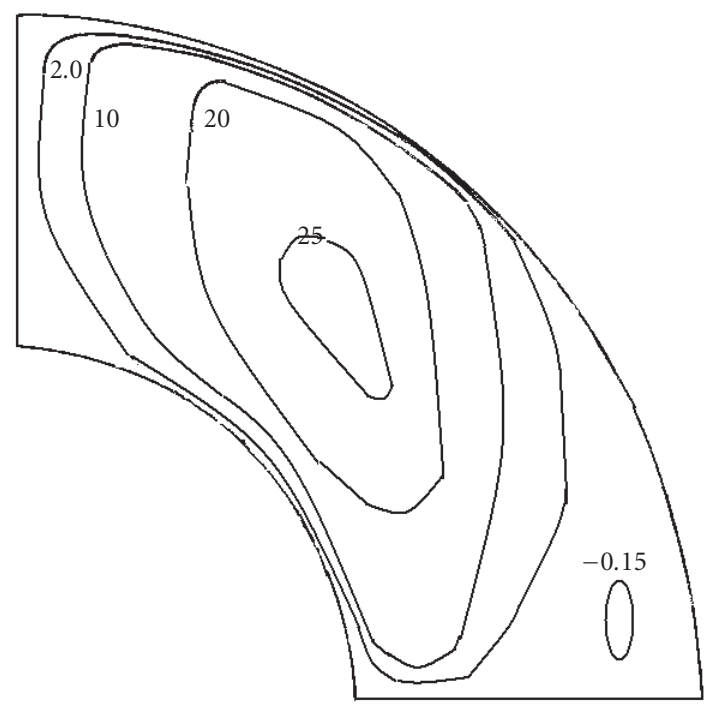

Figure 4.1. Level $\psi$ curves multiplied by $10^{4}$ for $\mathrm{Re}=400$.

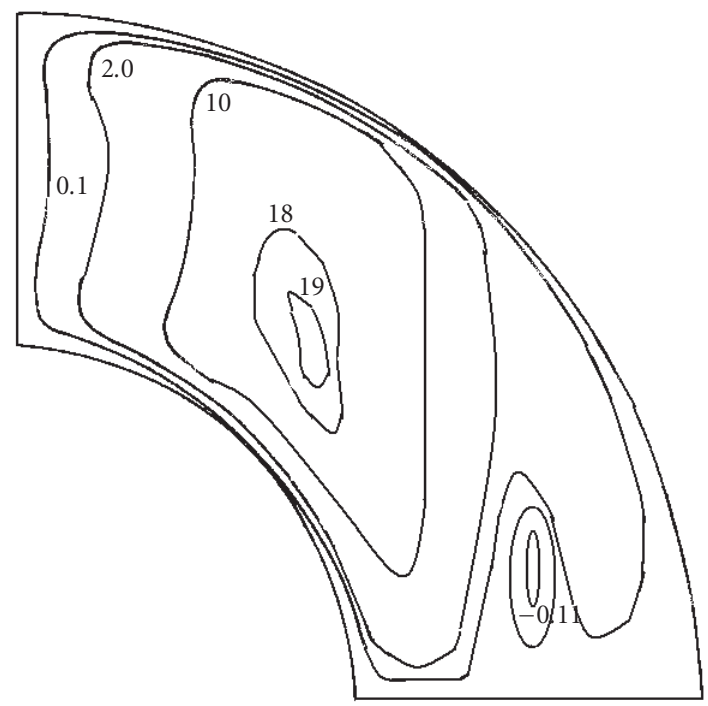

Figure 4.2. Level $\psi$ curves multiplied by $10^{4}$ for $\mathrm{Re}=1000$.

tends to a cylindrical sheath of radius approximately equal to that of the inner sphere. (See Figures 4.1 and 4.2.) As the Reynolds number is increased, this secondary flow pattern is maintained and no development of any disjoint closed curves is observed. When the Reynolds number is increased to 3000, a stable flow pattern is observed in the meridian plane with a change of shape. (See Figure 4.3). A recirculation region still exists near the equator but no visible signs of any disjoint close curves are observed as suggested by 


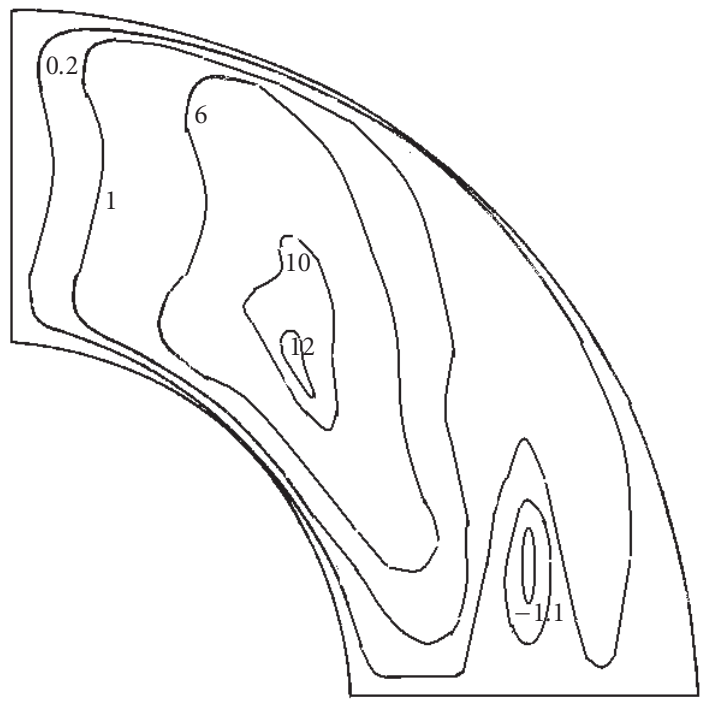

Figure 4.3. Level $\psi$ curves multiplied by $10^{4}$ for $\mathrm{Re}=3000$.

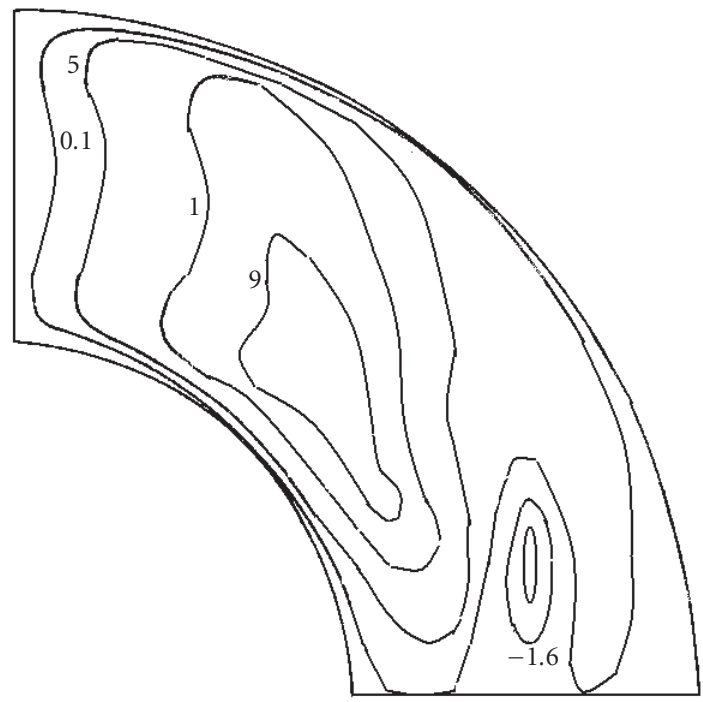

Figure 4.4. Level $\psi$ curves multiplied by $10^{4}$ for $\mathrm{Re}=5000$.

Schultz and Greenspan. For Re $=5000$, however, no comparison could be made with existing numerical results since existing methods are confined to flow smaller than or equal to 3000 due to instabilities in the iterative procedures in which they are based. Contour plots of the stream function for $\mathrm{Re}=5000$ are given in Figure 4.4. Our results are consistent with the flow visualization patterns obtained from experiments by Munson and Menguturk [9] as well as those of Wimmer [16]. 
Solutions were carried out for several values of $n s$ for each value of Re and some judgement was made as to the maximum value of $n s$ required. The solutions were terminated at $\operatorname{Re}=5000$, in which case we obtained convergence after the 20th-order approximation, $n s=20$, with a modified value for $Z(x)$. The solutions for $\mathrm{Re}=100$ was taken as the initial guess approximation. The effects of varying the grid size on the solutions were also investigated; typically, one chooses a grid size of $20 \times 40$. No significant changes in the solution occurred when the grid size was varied for various values of Re.

\section{References}

[1] E. L. Allgower and K. Georg, Continuation and path following, Acta Numerica, 1993, Acta Numer., Cambridge University Press, Cambridge, 1993, pp. 1-64.

[2] D. Greenspan, Numerical studies of steady, viscous, incompressible flow between two rotating spheres, Comput. \& Fluids 3 (1975), no. 1, 69-82.

[3] S. J. Liao, An analytic approximation of the drag coefficient for the viscous flow past a sphere, Internat. J. Non-Linear Mech. 37 (2002), no. 1, 1-18.

[4] - A direct boundary element approach for unsteady nonlinear heat transfer problems, Engineering Analysis with Boundary Elements 26 (2002), 55-59.

[5] - Beyond Perturbation: Introduction to the Homotopy Analysis Method, Chapman \& Hall/CRC Press, Florida, 2003.

[6] On the analytic solution of magnetohydrodynamic flows of non-Newtonian fluids over a stretching sheet, J. Fluid Mech. 488 (2003), 189-212.

[7] B. R. Munson and D. D. Joseph, Viscous incompressible flow between concentric rotating spheres. I: Basic flow, J. Fluid Mech. 49 (1971), 289-303.

[8] _ Viscous incompressible flow between concentric rotating spheres. II: Hydrodynamic stability, J. Fluid Mech. 49 (1971), 305-318.

[9] B. R. Munson and M. Menguturk, Viscous incompressible flow between concentric rotating spheres. III: Linear stability and experiments, J. Fluid Mech. 69 (1975), 705-719.

[10] M. Oettli, The homotopy method applied to symmetric eigenproblem, Ph.D. thesis, Swiss Federal Institute of Technology, Zurich, 1995.

[11] C. E. Pearson, A numerical study of the time dependent viscous flow between two rotating spheres, J. Fluid Mech. 28 (1967), 323-336.

[12] D. F. Roscoe, New methods for the derivation of stable difference representations for differential equations, J. Inst. Math. Appl. 16 (1975), no. 3, 291-301.

[13] D. Schultz and D. Greenspan, Improved solution of steady, viscous, incompressible flow between two rotating spheres, Comput. \& Fluids 7 (1979), no. 3, 157-163.

[14] W. Sha and K. J. Nakabayashi, On the structure and formation of spiral Taylor-Gortler vortices in spherical couette flow, J. Fluid Mech. 431 (2001), 323-345.

[15] F. M. White, Viscous Fluid Flow, McGraw-Hill, New York, 1991.

[16] M. Wimmer, Experiments on the stability of viscous flow between two concentric rotating spheres, J. Fluid Mech. 103 (1981), 117-131.

E. O. Ifidon: Department of Mathematics, University of Benin, PMB 1154, Benin City, Nigeria

E-mail address: ifidon@uniben.ng 


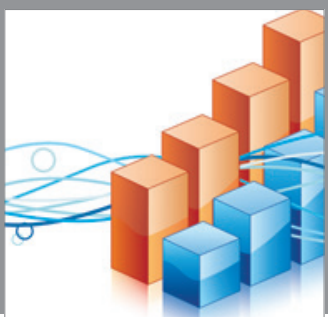

Advances in

Operations Research

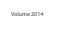

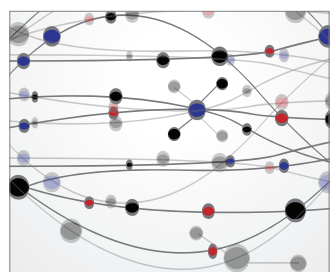

\section{The Scientific} World Journal
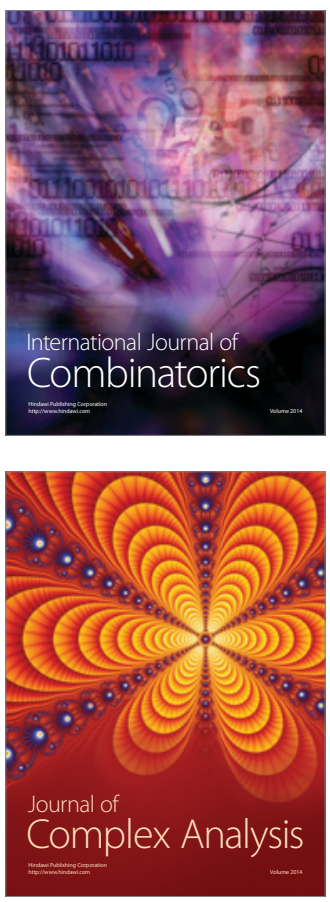

International Journal of

Mathematics and

Mathematical

Sciences
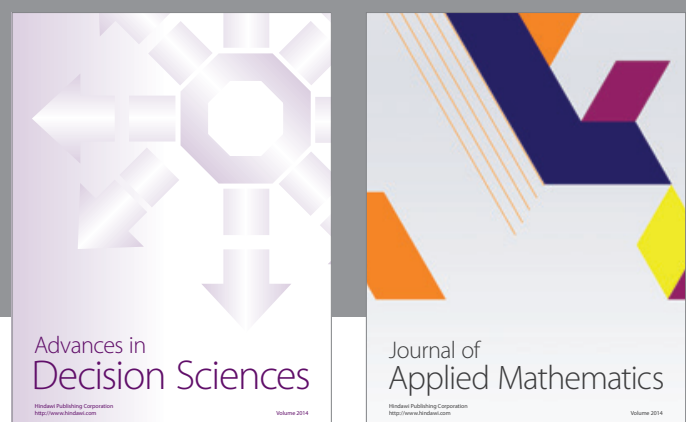

Journal of

Applied Mathematics
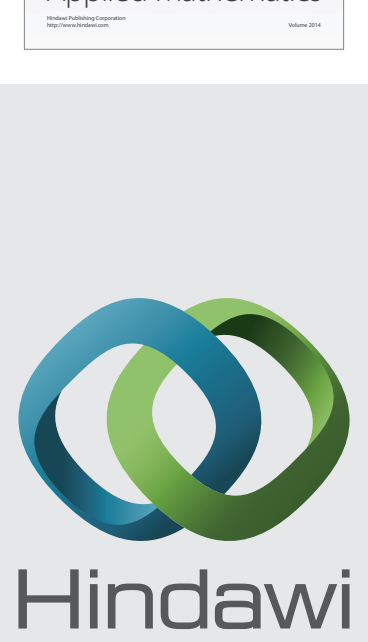

Submit your manuscripts at http://www.hindawi.com
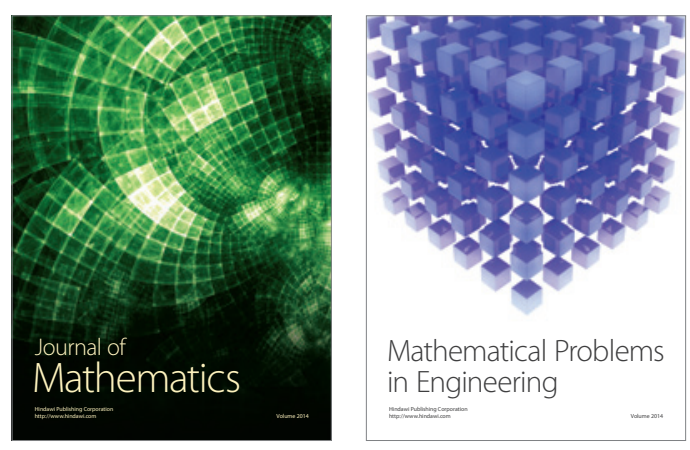

Mathematical Problems in Engineering


Journal of

Function Spaces
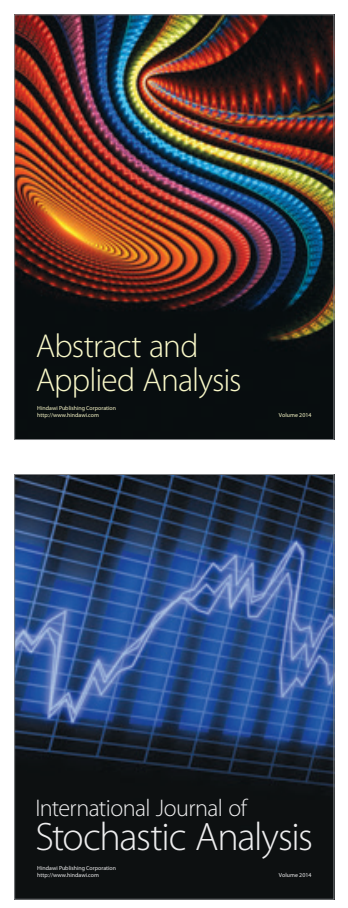

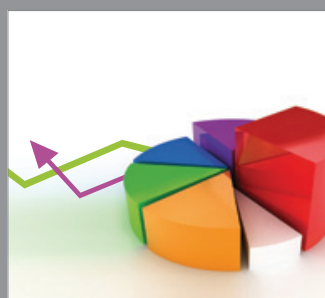

ournal of

Probability and Statistics

Promensencen
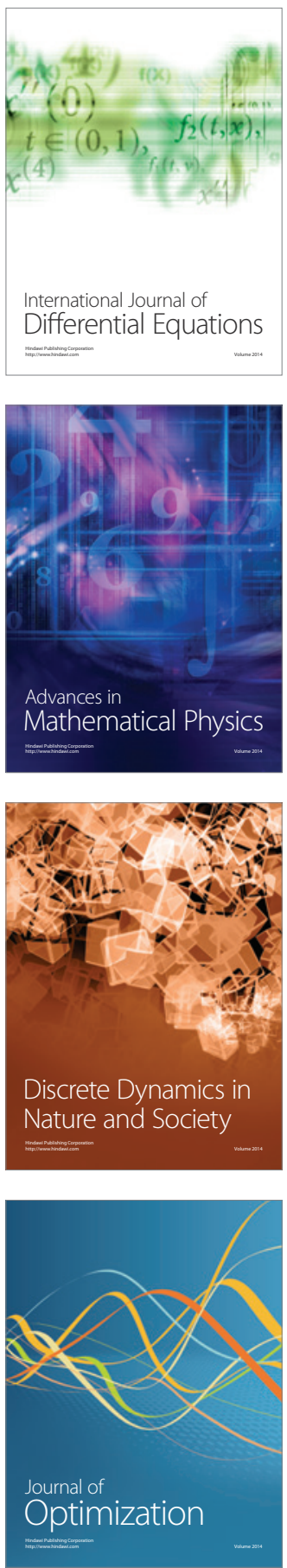